\title{
The hypoxia-inducible factor $\alpha$ pathway couples angiogenesis to osteogenesis during skeletal development
}

\author{
Ying Wang, ${ }^{1}$ Chao Wan, ${ }^{1}$ Lianfu Deng, ${ }^{1}$ Ximeng Liu, ${ }^{1}$ Xuemei Cao, ${ }^{1}$ Shawn R. Gilbert, ${ }^{1}$ \\ Mary L. Bouxsein, ${ }^{2}$ Marie-Claude Faugere, ${ }^{3}$ Robert E. Guldberg, ${ }^{4}$ Louis C. Gerstenfeld, ${ }^{5}$ \\ Volker H. Haase, ${ }^{6}$ Randall S. Johnson, ${ }^{7}$ Ernestina Schipani, ${ }^{8}$ and Thomas L. Clemens ${ }^{1,9}$ \\ 1Department of Pathology, University of Alabama at Birmingham, Birmingham, Alabama, USA. 'Department of Orthopedic Surgery, \\ Beth Israel Deaconess Medical Center, Boston, Massachusetts, USA. ${ }^{3}$ Department of Medicine, University of Kentucky, Lexington, Kentucky, USA \\ ${ }^{4}$ Woodruff School of Mechanical Engineering, Georgia Institute of Technology, Atlanta, Georgia, USA. ${ }^{5}$ Department of Orthopedic Surgery, \\ Boston University Medical Center, Boston, Massachusetts, USA. ${ }^{6}$ Department of Medicine, University of Pennsylvania School of Medicine, \\ Philadelphia, Pennsylvania, USA. ${ }^{7}$ Division of Biological Sciences, UCSD, San Diego, California, USA. ${ }^{8}$ Endocrine Unit, Massachusetts General Hospital \\ and Harvard Medical School, Boston, Massachusetts, USA. ${ }^{9}$ Veterans Administration Medical Center, Birmingham, Alabama, USA.
}

\begin{abstract}
Skeletal development and turnover occur in close spatial and temporal association with angiogenesis. Osteoblasts are ideally situated in bone to sense oxygen tension and respond to hypoxia by activating the hypoxiainducible factor $\alpha(\mathrm{HIF} \alpha)$ pathway. Here we provide evidence that HIF $\alpha$ promotes angiogenesis and osteogenesis by elevating VEGF levels in osteoblasts. Mice overexpressing HIF $\alpha$ in osteoblasts through selective deletion of the von Hippel-Lindau gene $(V b l)$ expressed high levels of $V e g f$ and developed extremely dense, heavily vascularized long bones. By contrast, mice lacking Hifla in osteoblasts had the reverse skeletal phenotype of that of the $\mathrm{Vbl}$ mutants: long bones were significantly thinner and less vascularized than those of controls. Loss of $\mathrm{Vbl}$ in osteoblasts increased endothelial sprouting from the embryonic metatarsals in vitro but had little effect on osteoblast function in the absence of blood vessels. Mice lacking both $\mathrm{Vhl}$ and Hifla had a bone phenotype intermediate between those of the single mutants, suggesting overlapping functions of HIFs in bone. These studies suggest that activation of the HIF $\alpha$ pathway in developing bone increases bone modeling events through cell-nonautonomous mechanisms to coordinate the timing, direction, and degree of new blood vessel formation in bone.
\end{abstract}

\section{Introduction}

The development of the mammalian skeleton takes place in distinct phases involving the initial migration of cells to the site of future bone, condensation of mesenchymal cells, and finally the differentiation of progenitors into chondrocytes and osteoblasts. During intramembranous bone formation, which gives rise to the flat bones of the skull, mesenchymal cells differentiate directly into bone-forming osteoblasts. By contrast, in endochondral bone formation, bones are formed through a 2 -stage mechanism that begins with the formation of a chondrocyte anlage, onto which osteoblasts then differentiate and deposit bone. Endochondral bone formation occurs in close spatial and temporal association and proximity to capillary invasion, suggesting that angiogenesis and osteogenesis are coupled.

The initial signals for blood vessel invasion into bone are unknown, but tissue hypoxia is believed to be critical for commencement of the angiogenic cascade (1). Hypoxia triggers the changes in oxygen-regulated gene expression via the activation of the Per/Arnt/Sim (PAS)

Nonstandard abbreviations used: ALP, alkaline phosphatase; ARNT, aryl hydrocarbon receptor nuclear translocator; Glut1, type 1 glucose transporter; HIF, hypoxiainducible factor; OC, osteocalcin; ODD, oxygen-dependent degradation domain; OPG, osteoprotegerin; Pgk, phosphoglycerate kinase; PHD1, prolyl hydroxylase 1; pVHL, von Hippel-Lindau protein; TRAP 5b, tartrate-resistant acid phosphatase form $5 \mathrm{~b} ; \mathrm{Vhl}$, von Hippel-Lindau; $\Delta H$ ifla mice, mice with conditional deletion of Hifla in osteoblasts; $\Delta V b l$ mice, mice with conditional deletion of $V b l$ in osteoblasts; $\Delta V b l$ $\Delta H i f 1 a$ mice, mice with conditional deletion of Vbl and Hifla in osteoblasts.

Conflict of interest: The authors have declared that no conflict of interest exists. Citation for this article: J. Clin. Invest. 117:1616-1626 (2007). doi:10.1172/JCI31581. subfamily of basic helix-loop-helix (bHLH) transcription factors (2). The hypoxia-inducible factors (HIFs) activate genes encoding proteins that mediate adaptive responses (e.g., angiogenesis) to reduced oxygen availability (3). The HIF complex consists of 1 of 3 $\alpha$ subunits (HIF- $1 \alpha$, HIF- $2 \alpha$, or HIF-3 $\alpha$ ) bound to the aryl hydrocarbon receptor nuclear translocator (ARNT), also known as HIF $\beta$. The level of HIF- $1 \alpha$ and HIF- $2 \alpha$ proteins is regulated by ongoing ubiquitination and proteasomal degradation following enzymatic prolyl hydroxylation on an oxygen-dependent degradation domain (ODD) (4). The E3 ligase von Hippel-Lindau protein (pVHL) binds directly to hydroxylated HIF $\alpha$ subunits and regulates their polyubiquitination and destruction by the proteasome (5). During hypoxia, prolyl hydroxylation is blocked, leading to HIF $\alpha$ stabilization, subsequent nuclear import, and dimerization with ARNT, which initiates the transcription of HIF-responsive genes (6).

As indicated above, formation of endochondral bone coincides with capillary ingrowth and angiogenesis. Furthermore, disruption of normal afferent blood supply, which occurs following bone fracture, leads to hypoxia of adjacent tissue. Based on these observations, we reasoned that cells of mesenchymal origin, including osteoblasts, are ideally positioned in bone to sense and respond to fluctuations in oxygen and nutrient supply. Consistent with this concept, osteoblasts and osteocytes respond to hypoxia by elevating the level of HIF $\alpha$, which in turn transactivates Vegf and other HIF target genes. We therefore hypothesize that osteoblasts use the HIF $\alpha$ pathway to sense reduced oxygen tension and transmit signals that impinge on angiogenic and osteogenic gene programs. 
A

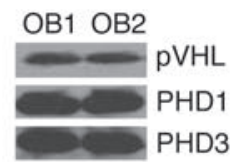

B

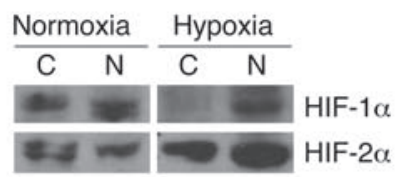

C

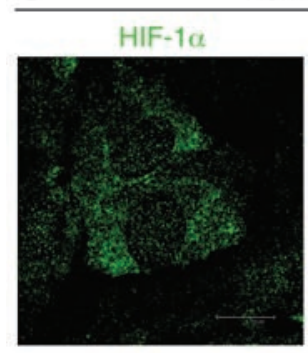

HIF-2 $\alpha$

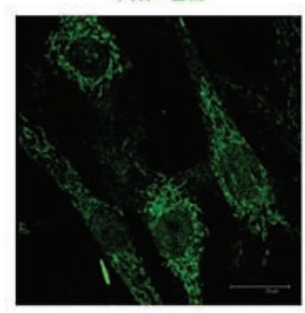

Normoxia

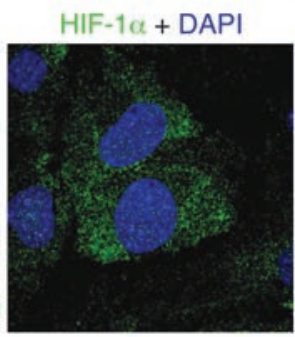

HIF $-2 \alpha+$ DAPI

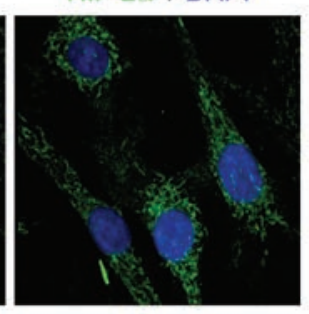

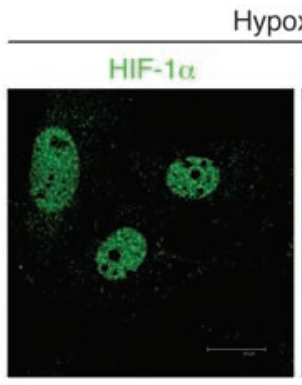

HIF-2Q

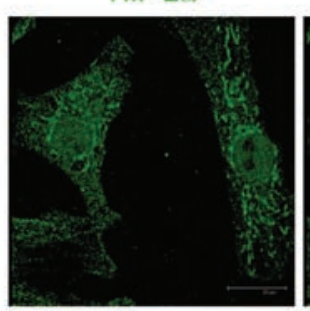

ypoxia

HIF-1 $\alpha$ + DAPI

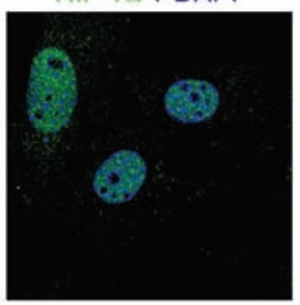

HIF- $2 \alpha+$ DAPI

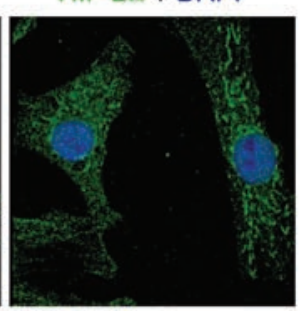

D

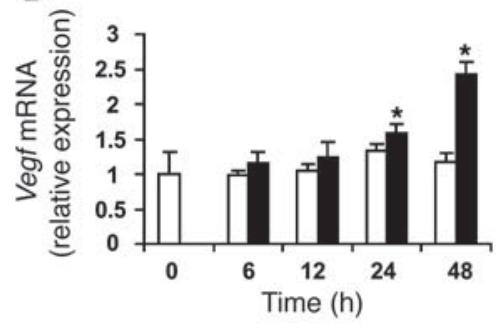

E

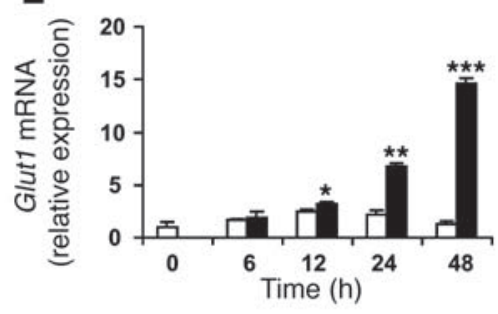

Figure 1

Primary mouse osteoblasts express components of the HIF $\alpha$ pathway. (A) Calvarial primary osteoblasts were obtained from wild-type mice and cultured in $\alpha$-MEM until confluent. Whole-cell lysate was analyzed by immunoblotting using antibodies against pVHL (top), PHD1 (middle), and PHD3 (bottom) in experiments performed in duplicate. OB, osteoblast. (B and C) Confluent cell monolayers were exposed to $21 \%$ (normoxia) or $2 \%$ (hypoxia) $\mathrm{O}_{2}$ for 24 hours. (B) Proteins from cytoplasm $(\mathrm{C})$ and nucleus $(\mathrm{N})$ were extracted separately and analyzed by immunoblotting with antibodies against HIF-1 $\alpha$ (top row) and HIF-2 $\alpha$ (bottom row). (C) Nuclear translocation of HIF-1 $\alpha$ (top row) and HIF-2 $\alpha$ (bottom row) was assessed by confocal microscopy as described in Methods. DAPI was used to stain the nuclei. Original magnification, $\times 100$. (D and E) Quantitative real-time PCR analysis was performed to determine Vegf and Glut1 mRNA expression after cell monolayers were exposed to normoxia (white bars) or hypoxia (black bars) for the indicated times. ${ }^{*} P<0.05 ;{ }^{* *} P<0.01$; ${ }^{* *} P<0.001$.

In this study, we used a genetic approach to determine the cellular and molecular effect of gain or loss of HIF function by conditional mutagenesis in osteoblasts during bone development. We show that constitutive activation of the HIF $\alpha$ pathway in mice promotes robust bone modeling and acquisition in long bones but not in the skull. This occurs through upregulation of Vegf and possibly other angiogenic factors primarily through cell- (osteoblast-) nonautonomous mechanisms. Conversely, loss of Hifla in osteoblasts results in narrow, less vascularized bones. These results suggest that activation of the HIF $\alpha$ pathway in osteoblasts during bone development couples angiogenesis to osteogenesis.

\section{Results}

Primary osteoblasts express components of the HIF $\alpha$ pathway. Oxygensensitive cells use the HIF $\alpha$ pathway to sense and respond to changes in ambient oxygen. As a first step in studying the role of HIFs in osteoblasts, we determined the expression of components of this pathway in primary mouse osteoblasts. Osteoblasts expressed abundant pVHL and prolyl hydroxylase 1 and 3 (PHD1 and -3) (Figure 1A). Antibodies against mouse PHD2 were not available at the time of this study. As shown in Figure 1, B and C, exposure of osteoblasts to $2 \% \mathrm{O}_{2}$ resulted in translocation of both HIF- $1 \alpha$ and HIF- $2 \alpha$ from the cytoplasm to the nucleus; nuclear translocation was more complete for HIF-1 $\alpha$ than for HIF-2 $\alpha$. Exposure of osteoblasts to hypoxia was associated with upregulation of HIF target genes Vegf and type 1 glucose transporter (Glut1) mRNA expression (Figure 1, D and E). Therefore, osteoblasts possess the major components of the HIF $\alpha$ pathway.

Mice lacking Vhl in osteoblasts have upregulated HIFa. To investigate the function of HIF $\alpha$ in bone development in vivo, we created genetic mouse models engineered for manipulation of the levels of HIF $\alpha$ in osteoblasts. In the first model, we generated mice that overexpressed $\mathrm{HIF} \alpha$ in osteoblasts by disrupting Vhl. Mice expressing the Cre recombinase driven by an osteocalcin (OC) promoter (OC-Cre) (7) were crossed with mice homozygous for the loxP-flanked (floxed) $V h l$ allele (hereafter designated as control) (8) to obtain mice lacking $V h l$ (hereafter designated $\Delta V h l$ ) and therefore overexpressing HIF $\alpha$ in osteoblasts. To determine the specificity of the $V b l$ deletion in osteoblasts, we performed allele-specific PCR using DNA isolated from selected tissues. These results demonstrated that Cre-mediated recombination of the floxed $\mathrm{Vhl}$ allele $(\Delta$ flox) occurred only in bone tissue (Figure 2A). Immunohistochemistry on fixed sections of distal femur showed upregulation of both HIF- $1 \alpha$ and HIF- $2 \alpha$ in osteoblasts lining bone surfaces of the $\Delta V h l$ mice (Figure 2B). Activation of the HIF $\alpha$ pathway in osteoblasts was also demonstrated in vitro following infection with adenoviral Cre to delete the floxed $\mathrm{Vbl}$ allele. pVHL levels were eliminated in both the cytoplasm and nucleus, which was associated with increased expression of both HIF- $1 \alpha$ and HIF- $2 \alpha$ proteins in the nuclear fraction (Figure $2 \mathrm{C}$ ). These changes 


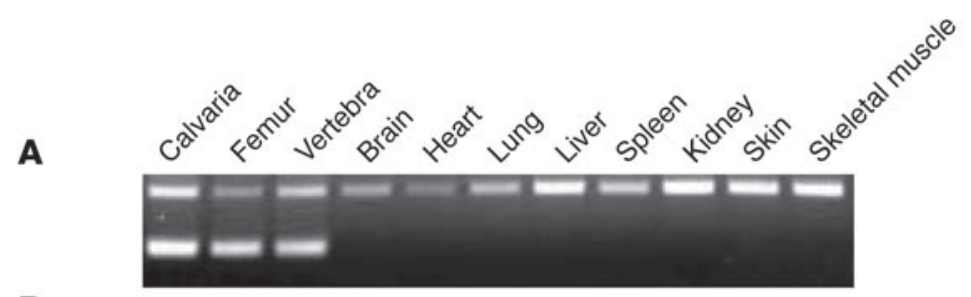

B

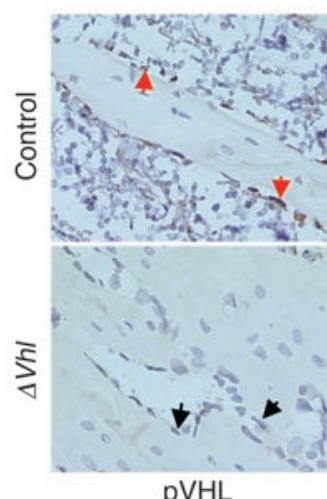

C Cytoplasm Nucleus

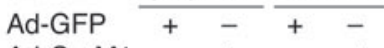

Ad-CreM1 - + -+

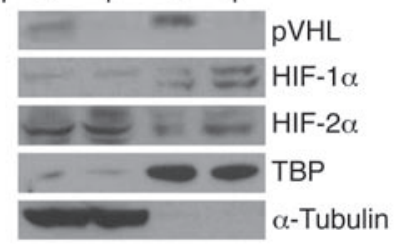

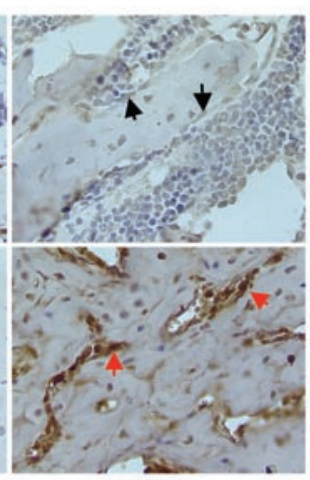

HIF-1 $\alpha$

\section{D}

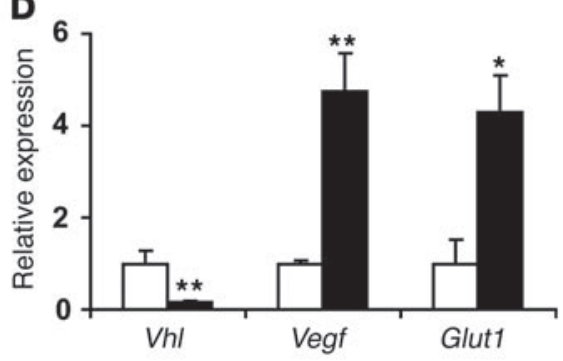

\section{Figure 2}

Osteoblast-specific, Cre-mediated deletion of $\mathrm{Vhl}$. (A) PCR analysis of Cre-mediated recombination in selected tissues from a $\Delta \mathrm{Vhl}$ mouse. The recombined allele ( $\Delta$ flox) was present exclusively in bone tissue. (B) Representative histological sections of distal femurs from 6-week-old control and $\Delta V h l$ mice after staining with antibodies against pVHL (left), HIF-1 $\alpha$ (middle), or HIF-2 $\alpha$ (right) as described in Methods. Sections were counterstained with hematoxylin. Red arrows indicate positive staining and black arrows negative staining in osteoblasts. Original magnification, $\times 400$. (C and D) Confluent monolayers of $\mathrm{Vhl}$ floxed primary osteoblasts were infected with either Ad-GFP or Ad-CreM1 (100 MOI) for 48 hours. (C) Proteins in the cytoplasm and nucleus were extracted separately and analyzed by immunoblotting with antibodies against $\mathrm{pVHL}, \mathrm{HIF}-1 \alpha$, and HIF-2 $\alpha$. Immunoblots for TBP and $\alpha$-tubulin were used as loading controls for nuclear and cytoplasmic proteins, respectively. TBP, TATA box-binding protein. (D) Total mRNA was extracted from confluent monolayers of osteoblasts 48 hours after adenoviral infection, and gene expression for Vhl, Vegf, and Glut1 was determined by quantitative real-time PCR. White bars represent Ad-GFP infection; black bars represent Ad-CreM1 infection. ${ }^{\star} P<0.05$; ${ }^{* \star} P<0.01$. were accompanied by increased expression of mRNA for the HIF target genes Vegf and Glut1 (Figure 2D). Expression of transcripts for all 3 Vegf isoforms ( $\operatorname{Veg} f_{120}, \operatorname{Veg} f_{164}$, and $\left.V e g f_{188}\right)$ was equally upregulated following $V h l$ deletion (Supplemental Figure 1; supplemental material available online with this article; doi:10.1172/JCI31581DS1).

Loss of Vhl in osteoblasts increases long bone volume. $\Delta \mathrm{Vbl}$ mice were born at the expected mendelian ratios and were similar in size and weight to control littermates. $\mu \mathrm{CT}$ of long bones revealed striking and progressive increases in bone volume in mutant femurs compared with wild-type controls (Figure 3, A and B). To quantitatively assess bone turnover, static and dynamic histomorphometric analyses were performed on femurs from both $\Delta V h l$ mice and control littermates at 3 weeks of age, when bone modeling in the mouse is very active (7). Consistent with the $\mu \mathrm{CT}$ results, bone volume was significantly increased $(P=0.014$; Figure $3 C)$, whereas trabecular separation was greatly reduced $(P=0.008$; Figure $3 \mathrm{D})$ in $\Delta V b l$ mice. However, at this time, neither the number of osteoblasts (Supplemental Figure 2A) nor the rate of bone formation (Figure 3E) was elevated relative to controls, suggesting that the increased bone volume in $\Delta V b l$ mice might have occurred earlier. To investigate this possibility, histomorphometric analysis was performed on femurs from 7-day-old $\Delta V b l$ and control mice. At this time, double calcein labeling showed an accelerated bone formation rate in the mutant mice compared with controls (Figure 3F). In addition, osteoblast number was significantly increased in the 7-day-old $\Delta V b l$ mice compared with controls $(P=0.039$; Figure $3 \mathrm{G})$. Thus, these results demonstrate that bone modeling is increased in $\Delta V b l$ mice as early as 7 days of age. To exclude the possibility that the greatly increased trabecular bone volume was masking an absolute increased osteoblast number in $\Delta V b l$ mice, we measured osteoblast numbers per tissue area in $\Delta V b l$ and control mice at 3 (Supplemental Figure 2B) and 6 weeks of age (data not shown). Osteoblast numbers calculated per tissue area were not significantly altered in $\Delta V b l$ bone. As an additional measure of osteoblast activity, we assayed the level of serum OC in $\Delta V b l$ and control mice. These results show a slight and insignificant decrease in OC in the Vhl mutants (Supplemental Figure 2C). Taken together, these data demonstrate that bone modeling is increased in $\Delta V b l$ mice within the first week of life but appears to decline at latter stages of postnatal development.

The increased accumulation of bone seen in the mutant animals could theoretically be due to defective bone resorption, which would be expected to be associated with decreased number and activity of osteoclasts. However, the number of osteoclasts expressed either as number per bone surface or number per total tissue area was not significantly different from that of controls at 3 weeks (Supplemental Figure 3, A and B). Moreover, the serum level of osteoprotegerin (OPG), an osteoclast inhibitory cytokine, was similarly unaffected in the mutants (Supplemental Figure 3C). Therefore, these data suggest that the increased bone volume in the $\mathrm{Vbl}$ mutants resulted primarily from increased numbers and activity of osteoblasts at an early age. Indeed, serum levels of osteoclastderived tartrate-resistant acid phosphatase form 5b (TRAP 5b) were modestly decreased in 6-week-old $\Delta V h l$ mice (Supplemental Figure 3D). These results suggest that bone turnover declines in 
A

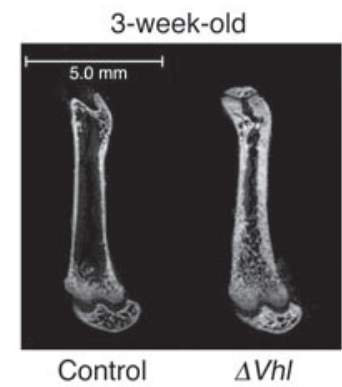

B

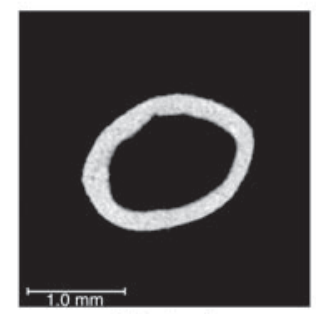

Control

E

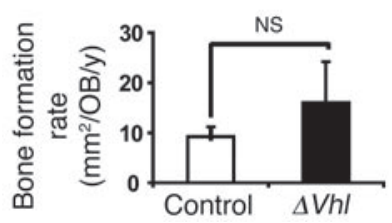

6-week-old
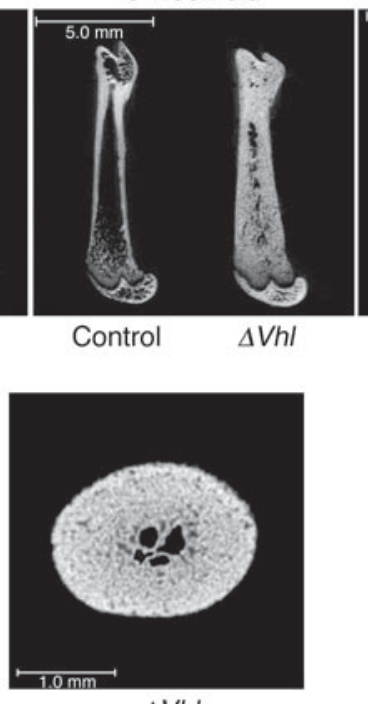

F

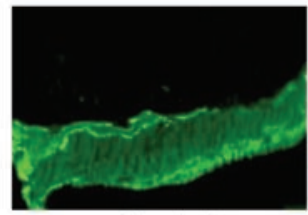

Control

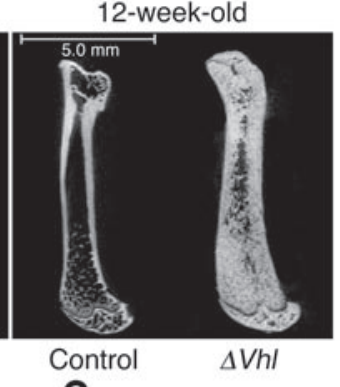

C
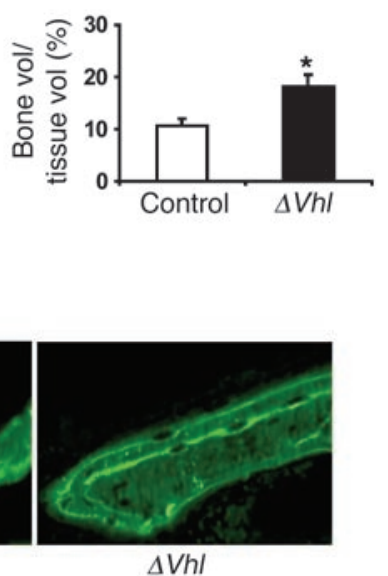

G

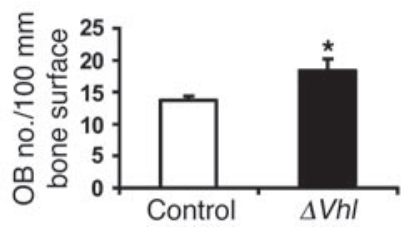

Figure 3

Disruption of $V h l$ in osteoblasts increases long bone volume. (A) Representative $\mu \mathrm{CT}$ images of the femurs from $\Delta V h l$ and control mice at the age of 3, 6, and 12 weeks. Scale bars: $5.0 \mathrm{~mm}$. (B) Representative femoral cross sections from 6-week-old $\Delta V h /$ and control mice. Scale bars: $1.0 \mathrm{~mm}$. (C-E) Histomorphometric analyses were performed on femoral sections from $\Delta V h /$ mice and controls at 3 weeks of age as described in Methods. Comparisons of trabecular bone volume (C), trabecular separation (D), and bone formation rate per osteoblast (E) in control (white bars; $n=6$ ) and $\Delta V h /$ mice (black bars; $n=7$ ) are shown. Data represent mean \pm SEM. ${ }^{*} P<0.05$; ${ }^{*} P<0.01$. (F) Seven-day-old mice were labeled with sequential doses of calcein before sacrifice. Representative calcein-labeled sections of distal femur from control and $\Delta \mathrm{Vh} / \mathrm{mice}$ are shown. Original magnification, $\times 400$. (G) Quantitative histomorphometric measurement of osteoblast number was performed at the distal femur of 7-day-old $\Delta \mathrm{Vhl}$ (black bar; $n=3$ ) and control mice (white bar; $n=3$ ). Data represent mean $\pm \mathrm{SEM}$. ${ }^{*} P<0.05$.

the mature $\Delta V b l$ mice, accounting at least in part for the progressive increase in postnatal bone acquisition.

Overexpression of HIF in osteoblasts does not affectcalvarialboneformation. As indicated above, the mechanisms responsible for formation of the flat bones of the skull (intramembranous ossification) are different than those that form the long bones (endochondral ossification). Surprisingly, mice lacking $V h l$ had no detectable changes in calvarial bone morphology. Gross $\mu \mathrm{CT}$ and histological analysis showed that cranial bone was similar in control and $\Delta V b l$ mice (Figure 4A). Quantitative histomorphometric analysis showed that bone volume (Figure 4B) and osteoblast numbers (Figure 4C) in the $\Delta V b l$ mice were not significantly different from those in controls. The lack of effect of the $\mathrm{Vbl}$ mutation on calvarial bone was not due to incomplete excision of the $\mathrm{Vhl}$ gene in this tissue (Figure 2A). Therefore, these results suggest that overexpression of HIF $\alpha$ in mouse osteoblasts appears to preferentially promote the acquisition of bone formed by endochondral processes.

Increased long bone volume in $\Delta V h l$ mice is associated with increased angiogenesis and Vegf production. At necropsy, we noted that the long bones from the $\mathrm{Vbl}$ mutants were more richly perfused with blood compared with control bones (Figure 5A), suggesting that deletion of $\mathrm{Vhl}$ may affect angiogenesis during bone development. To directly assess the impact of the Vbl mutation on bone vasculature we performed contrast-enhanced $\mu \mathrm{CT}$ imaging. $\mu \mathrm{CT}$ imaging of vasculature in Microfil-perfused bones revealed a striking increase in the density of the vasculature in $\Delta V b l$ as early as 7 days of age, with progressively increased vessel numbers with age (Figure 5B). Quantitative analysis showed that vessel surface and volume were both increased in $\Delta V h l$ mice relative to controls (Figure 5, C and D). These observations suggest that the loss of $V h l$ with consequent upregulation of HIF $\alpha$ in osteoblasts increases the production of angiogenic factors that promote formation of the bone vasculature. Consistent with this idea, the expression of 2 HIF target genes, Vegf (Figure 5E) and phosphoglycerate kinase (Pgk) (Supplemental Figure $4 \mathrm{~A}$ ), was upregulated in trabecular bone of $\Delta V b l$ femurs. The serum level of VEGF was not elevated in $\Delta V h l$ mice, as determined by ELISA (Supplemental Figure 4B), indicating that HIF $\alpha$-mediated upregulation of VEGF only occurred locally in bone.

To further investigate the role of VEGF in promoting angiogenesis in the $\Delta V h l$ mice, we performed an angiogenesis assay using explants of E17.5 mouse metatarsals. Control metatarsals exhibited a small degree of endothelial sprouting (Figure 5F), which was greatly enhanced by treatment with a recombinant VEGF (Figure 5G). By contrast, endothelial sprouting from $\Delta V h l$ metatarsals was 
A

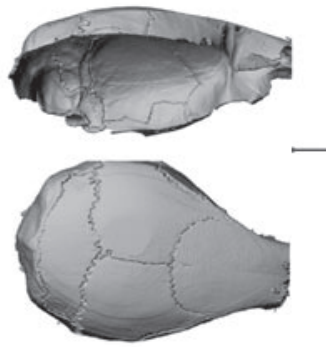

Control

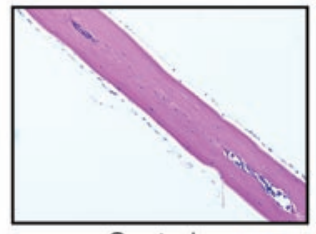

Control

\section{B}

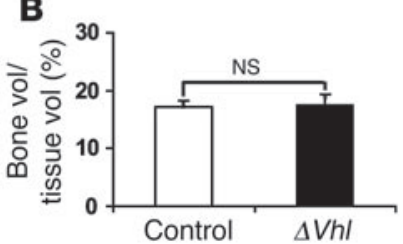

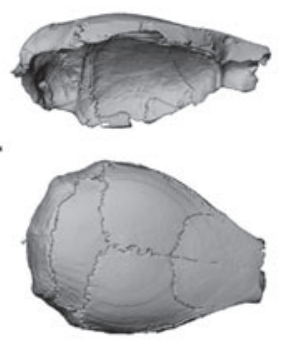

$\Delta \mathrm{Vhl}$

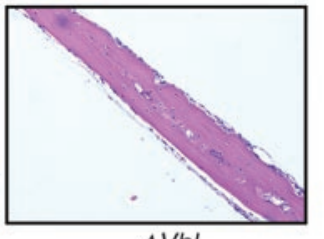

$\Delta V h l$
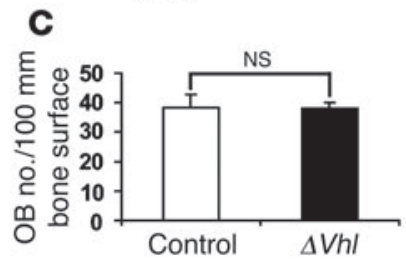

much greater than that from controls and was virtually abolished by preincubation with a VEGF-neutralizing antibody (Figure 5I), but not with a control IgG (Figure $5 \mathrm{H}$ ). This finding suggests that VEGF is upregulated in the long bones of the $\Delta \mathrm{Vbl}$ mice and contributes to increased angiogenesis in bone tissue.

Upregulation of HIF $\alpha$ in osteoblasts independent of angiogenesis does not alter osteoblast proliferation and apoptosis. The results described above suggested that increased long bone volume in $\Delta V h l$ mice occurred in part through cell-nonautonomous effects on angiogenesis. To investigate possible cell- (osteoblast-) autonomous effects of HIF $\alpha$ overexpression on the osteoblast performance independent of angiogenesis, we determined the effect of HIF $\alpha$ overexpression on osteoblast proliferation, survival, and differentiation following disruption of $V b l$ with adenovirally driven Cre. Deletion of $\mathrm{Vbl}$ in this manner resulted in the desired upregulation of HIF-1 $\alpha$ and HIF-2 $\alpha$ (Figure 2C) but had no significant effect on proliferation and apoptosis as assessed using flow cytometry of BrdU- (Figure 6, A and B) and annexin V-stained cells (Figure 6, $\mathrm{C}$ and $\mathrm{D}$ ). We next determined the effect of HIF $\alpha$ upregulation on osteoblast differentiation by growing cells in ascorbate-containing medium and staining for alkaline phosphatase (ALP) and von Kossa to assess bone mineral deposition. Disruption of $V b l$ had no appreciable effect on ALP expression and only slightly increased calcified nodule formation relative to that of control cells infected with a GFP virus (Figure 6, E-G). Moreover, the expression of runt-related transcription factor $2(R u n \times 2)$ and $O C$, markers for early and late osteoblast differentiation, respectively, was not significantly altered in the Vbl-deficient cells (Figure 6H). These results suggest that loss of $\mathrm{Vbl}$ and upregulation of HIF $\alpha$ in osteoblasts have minimal effects on in vitro osteoblast performance and further suggest that overexpression of HIF $\alpha$ in osteoblasts in vivo promotes bone formation primarily by cell-nonautonomous effects on angiogenesis.

\section{Figure 4}

Loss of $V h l$ in osteoblasts does not alter calvarial bone. (A) Representative $\mu \mathrm{CT}$ images of calvaria from 12-week-old $\Delta \mathrm{Vhl}$ and control mice (top). Scale bar: $2.0 \mathrm{~mm}$. The bottom panels show H\&E-stained sections of calvaria from 6 -week-old control and $\Delta V h /$ mice. Original magnification, $\times 100$. (B and C) Quantitative histomorphometric measurement of bone volume and osteoblast number was performed on calvarial sections from 6-week-old $\Delta V h l$ (black bars; $n=3$ ) and control mice (white bars; $n=3$ ). Data represent mean \pm SEM.

Inactivation of Hif1 a in osteoblasts decreases bone volume and vascularity. To definitively establish the role of HIFs in osteoblasts, we created a second mouse model lacking Hif1a in osteoblasts ( $\Delta$ Hif1a) by crossing the OC-Cre mouse (7) with a mouse carrying a conditional, floxed Hifla allele (control) (9). Mice lacking Hifla in osteoblasts were viable and developed normally. Allele-specific PCR analysis on tissues from these animals showed that recombination of the floxed Hifla allele $(\Delta$ flox $)$ occurred exclusively in bone tissue (Figure $7 \mathrm{~A})$. Immunohistochemistry confirmed the loss of HIF- $1 \alpha$ protein and increased HIF-2 $\alpha$ expression in osteoblasts from $\Delta H$ ifla mice (Figure 7B). $\mu \mathrm{CT}$ images at the mid-shaft of the femur showed that the diameter of the mutant bones was reduced relative to that of controls (Figure 7C). Histomorphometric analysis at this site revealed a significant reduction in the osteoid volume along with the reduced number of osteoblasts in the mutant bones (Supplemental Figure 5, A and B). $\mu$ CT analysis of Microfil-perfused bones from 3-week-old mice showed a reduction in vascular density in $\Delta H$ ifla mice compared with controls (Figure 7D). Thus, loss of Hifla in osteoblasts appears to result in thinner, less vascularized long bones and produces a reverse bone phenotype to that of the $\Delta V b l$ mice. Even so, the reduction in bone and blood vessel volume in these animals was less pronounced than we anticipated and suggested that other factors may function redundantly with HIF- $1 \alpha$ in bone tissue. Since HIF- $2 \alpha$ and HIF- $1 \alpha$ are known to exert overlapping functions in a number of tissues, we investigated the effect of deletion of Hifla on HIF- $2 \alpha$ expression using primary mouse osteoblasts in vitro. Introduction of Cre-expressing adenovirus into Hifla floxed primary osteoblasts knocked down the levels of HIF- $1 \alpha$ and increased HIF- $2 \alpha$ protein levels (Figure 7E). These changes were associated with upregulation of Hif $2 a$ and Vegf mRNA levels (Figure 7F). Based on these results, we conclude that deletion of Hifla in osteoblasts leads to compensatory increases in expression of HIF-2 $\alpha$, which can partially substitute for the loss of HIF-1 $\alpha$ function.

To further investigate the role of HIF- $1 \alpha$ in postnatal bone formation, we created a third mouse model lacking both Vhl and Hifla in osteoblasts $(\Delta V b l / \Delta H i f 1 a)$ by crossbreeding the $\Delta V b l$ and $\Delta H i f 1 a$ mice. If HIF- $1 \alpha$ functions independently of HIF- $2 \alpha$ or other factors to promote angiogenesis and osteogenesis, then mice lacking both $\mathrm{Vbl}$ and Hifla should exhibit a bone phenotype similar to that of the $\Delta H$ ifla mice. However, 6-week-old double-mutant mice had significantly increased cortical (Figure 8A) and trabecular bone volume (data not shown) relative to control littermates. The bone volume in the double mutants remained lower than that seen in the $\Delta V b l$ single knockout mice (compare Figure 8A with Figure 3B). By contrast, vascular densities were greatly increased in the double-mutant bones and were similar to those seen in the $\Delta V b l$ mice (Figure $8 \mathrm{~B}$ ). This result suggested that the lack of both $\mathrm{Vhl}$ and Hifla caused upregulation of HIF- $2 \alpha$ abundance and function. In agreement with this idea, osteoblasts lining trabecular surfaces of the double mutants had increased HIF-2 $\alpha$ immunoreactivity compared with controls (Figure $8 \mathrm{C}$ ) and 
A

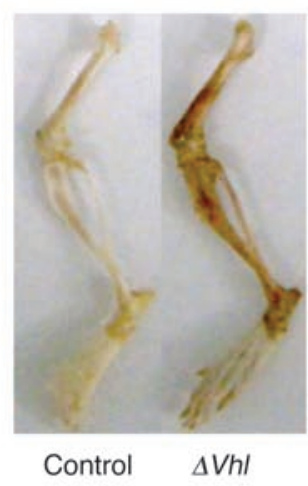

B

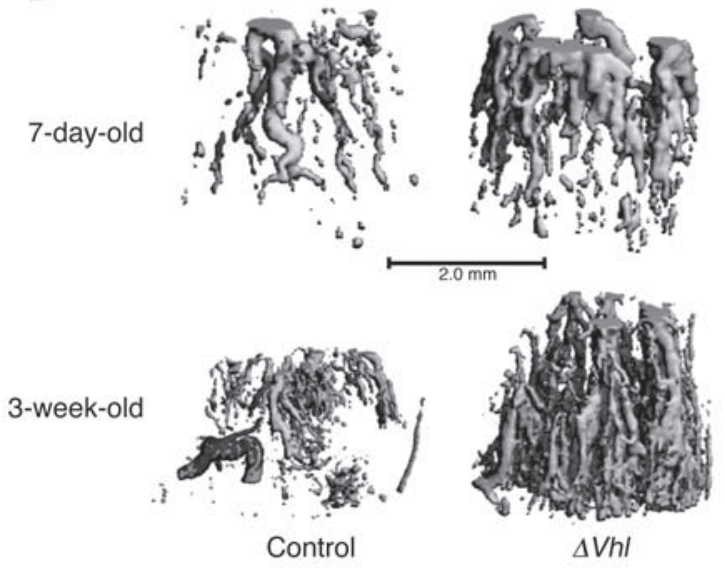

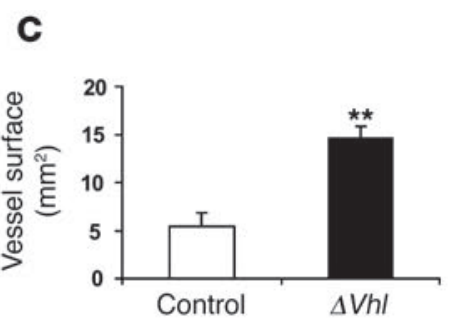

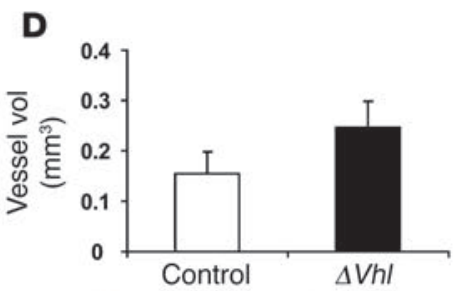

E

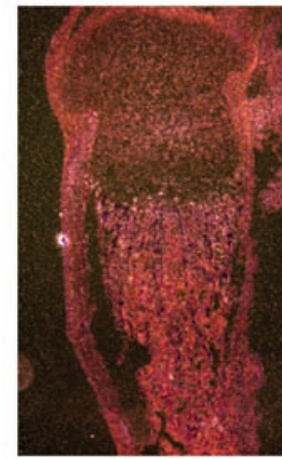

Control

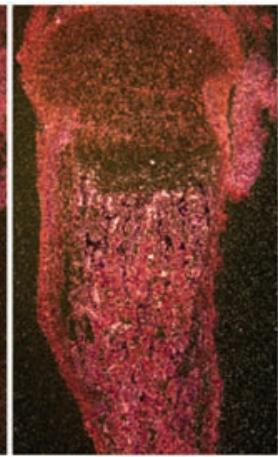

$\Delta V h l$
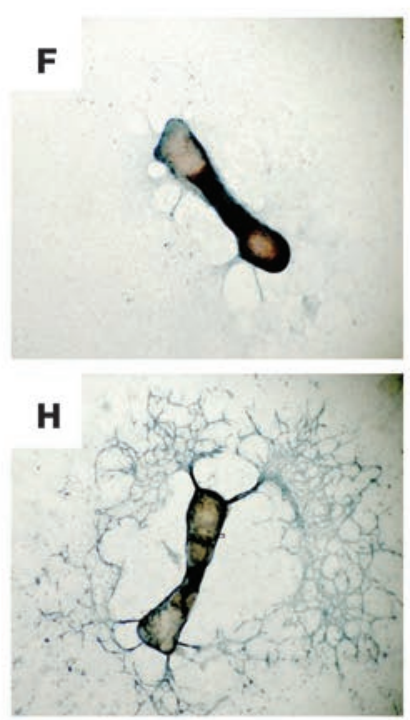

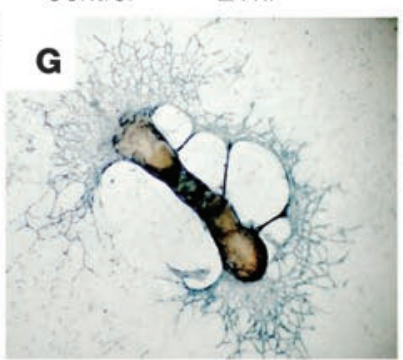

I

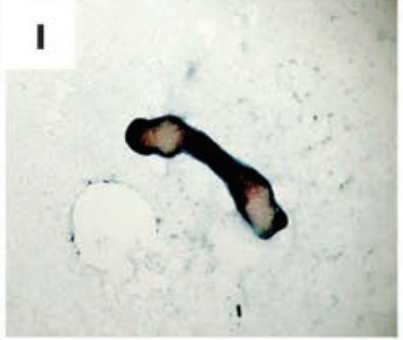

\section{Figure 5}

Increased angiogenesis in long bones of $\Delta V h /$ mice. (A) Photograph of hind limbs from $\Delta V h /$ and control mice. (B) Representative $\mu C T$ images of vasculature in Microfil-perfused femurs from 7-day-old and 3-week-old $\Delta V \mathrm{hl}$ and control mice. Scale bar: $1.0 \mathrm{~mm}$. (C and D) Morphological analysis of vessel surface and volume within femurs from Microfil-perfused $\Delta V h l$ (black bars; $n=3$ ) and control (white bars; $n=3$ ) mice. Data represent mean \pm SEM. ${ }^{* *} P<0.01$. (E) In situ hybridization analysis with Vegf mRNA on histological sections from 3-day-old control and $V h /$ mutant femurs. Original magnification, $\times 40$. (F-I) In vitro angiogenesis assay. Metatarsals were dissected from control and $\Delta V h / \mathrm{E} 17.5$ fetuses and cultured in $\alpha$-MEM for 14 days. The assay was performed using anti-CD31 antibody as described in Methods. Representative images are shown. Original magnification, $\times 25$. (F) Little detectable endothelial sprouting from control metatarsal. (G) Massive endothelial sprouting in control metatarsal treated with recombinant VEGF $(10 \mathrm{ng} / \mathrm{ml})$. (H) Extensive endothelial sprouting from $\Delta V h /$ metatarsal remained intact after treatment with a mouse control lgG (100 ng/ml). (I) Specific inhibition of endothelial sprouting in $\Delta V \mathrm{hl}$ metatarsal using a VEGF-neutralizing antibody (100 $\mathrm{ng} / \mathrm{ml}$ ). Data are representative of 3 independent experiments.

elevated Vegf mRNA expression (Figure 8D). Taken together, these results suggest that in the absence of HIF- $1 \alpha$, HIF-2 $\alpha$ functions like HIF- $1 \alpha$ to enhance VEGF production and drive angiogenesis.

\section{Discussion}

In this article, we present genetic evidence that the HIF $\alpha$ pathway is critical for coupling angiogenesis to osteogenesis during long bone formation. Activation of HIF $\alpha$ in osteoblasts through disruption of its degradation pathway produced robust bone modeling early in development. We attribute the increased bone formation to the increased angiogenesis, which appears to be mediated primarily by VEGF produced by the HIF $\alpha$-overexpressing osteoblasts. Conversely, disruption of Hifla in the osteoblast produced the reverse phenotype, i.e., thinner and less vascularized bones, clearly demonstrating the importance of the HIF $\alpha$ pathway in osteoblast-driven bone formation.

The development of the skeletal elements occurs in close association with and proximity to capillary ingrowth. The importance of a vascular supply in skeletogenesis was first demonstrated experimentally by Trueta and colleagues $(10,11)$, who demonstrated that bone mineralization and the expansion of the hypertrophic zone in the growth plate were decreased following the interruption of the blood supply to the growth plate. More contemporary studies by Gerber and Ferrara showed that impairment of angiogenesis decreased trabecular bone formation as well as the expansion of the hypertrophic zone into the growth plate (12). Moreover, it is known that the rate of bone formation and blood flow in the 
A
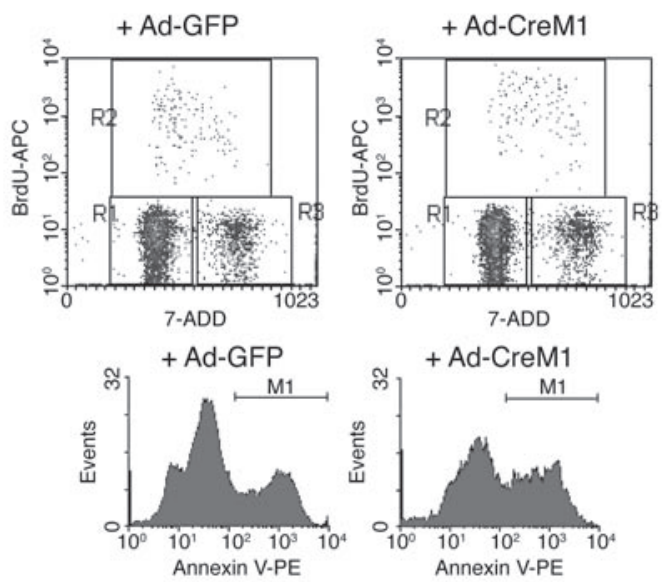

E
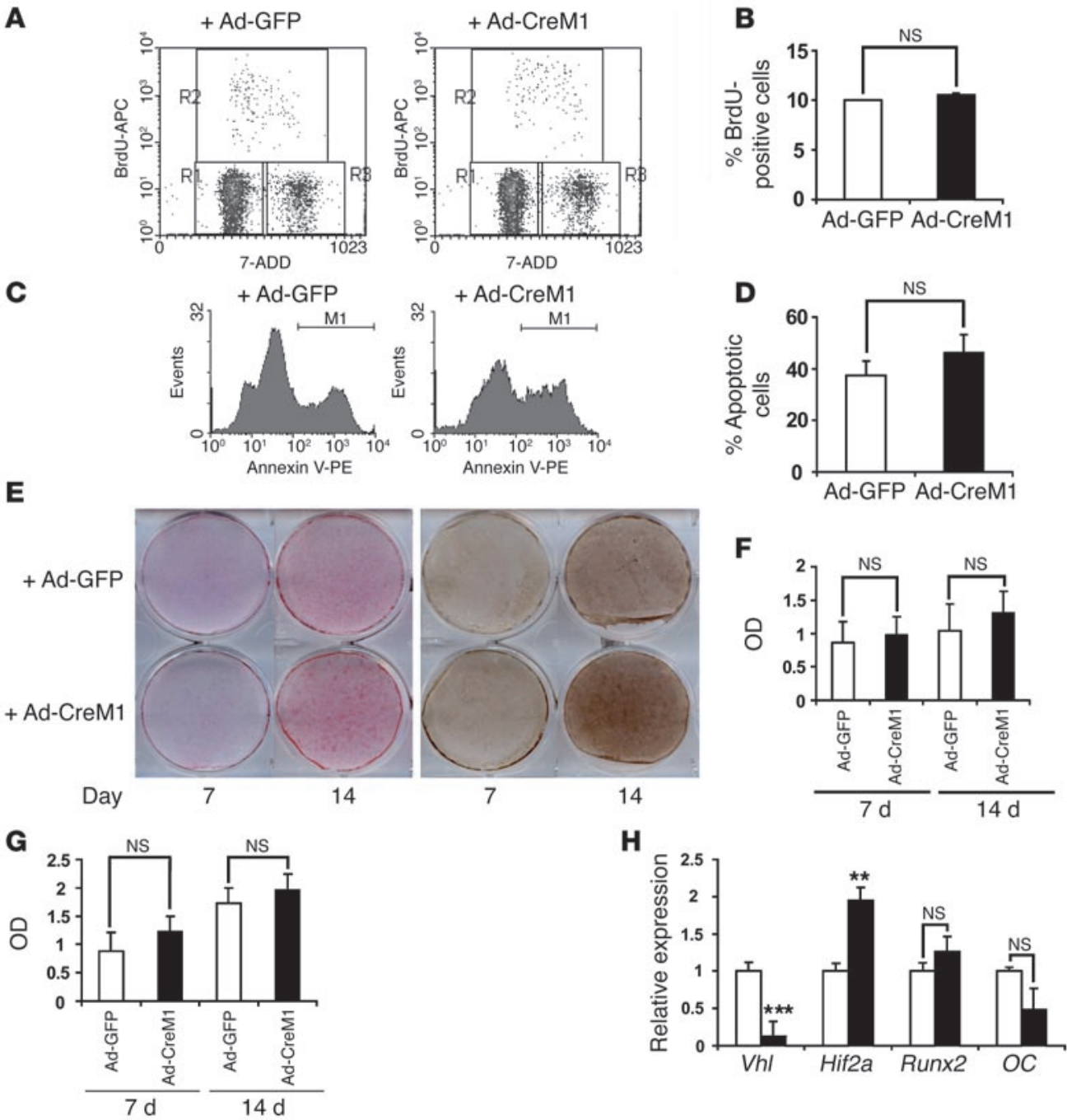

H

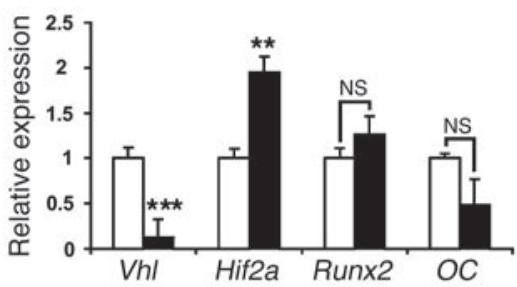

Figure 6

Deletion of $\mathrm{Vhl}$ in primary osteoblasts in vitro does not affect osteoblast proliferation and apoptosis. Confluent $\mathrm{Vhl}$ floxed primary osteoblast monolayers were infected with either adenoGFP or adeno-CreM1 (100 MOI). $\mathrm{Vhl}$ mRNA expression in infected osteoblasts was determined by real-time PCR 48 hours after infection to assess deletion efficiency. Cell proliferation, apoptosis, and differentiation assays were performed as described in Methods. (A and $\mathbf{B}$ ) Cell proliferation was assessed by flow cytometry using BrdU incorporation. (C and D) Cell apoptosis was assessed by flow cytometry using annexin V-PE staining. (E) Mineralized nodule formation was determined by ALP (left) and von Kossa staining (right) 7 and 14 days after cells were cultured in osteogenic medium. ( $\mathbf{F}$ and $\mathbf{G}$ ) Densitometric analysis of ALP and von Kossa staining observed in E using NIH ImageJ 1.36b. Data represent mean \pm SEM. (H) Measurement of $\mathrm{Vhl}$, Hif1a, runt-related transcription factor 2 (Runx2), and OC mRNA expression by quantitative real-time PCR at day 14 of osteogenic induction. ${ }^{\star \star} P<0.01$; ${ }^{* * *} P<0.001$ mature animal are tightly coupled (13). In this regard, the circulation is believed to be the major source for osteoclast precursors and the delivery of growth factors to individual remodeling units. Hauge et al. have suggested the existence of a discrete anatomical structure that they termed the bone remodeling compartment, which would serve to regulate bone remodeling $(14,15)$. Cells lining this structure express mediators of osteoclastogenesis including OPG and receptor activator of NF-кB ligand (RANKL), consistent with a role in coupling bone formation and resorption (15). Together, these observations support the hypothesis that blood vessel invasion is critical for bone formation and turnover and support the existence of anatomical and molecular mechanisms (e.g., $\mathrm{HIF} \alpha$ ) that coordinate angiogenesis and osteogenesis.

The results presented herein demonstrate that activation of the HIF $\alpha$ pathway preferentially influenced modeling of the endochondral bones. As reviewed above, endochondral bones form through a process whereby cells from the paraxial mesoderm and lateral plate mesoderm differentiate into chondrocytes to form an avascular cartilage anlage. This template is subsequently vascularized and then converted into bone. During this time, angiogenesis is essential for the normal differentiation of chondrocytes and appropriate alignment of the chondrocytes in the growth plate. Indeed, previ- ous studies by Schipani et al. have shown that the HIFo pathway is required for the normal endochondral growth plate development. In their model, chondrocytes lacking Hifla in the center of both the proliferative and the upper hypertrophic zones underwent programmed cell death, which caused disorganization of chondrocyte palisades and led to the misshaped growth plates (9). However, in contrast to the findings of the present study, constitutive activation of HIF- $1 \alpha$ in chondrocytes though disruption of $V h l$ had no apparent effect on angiogenesis but instead produced cell-autonomous effects that impacted survival and function of chondrocytes (16).

A striking observation in the current studies was that manipulation of the HIF $\alpha$ levels in osteoblasts did not influence the formation of the flat bones of the skull. These bones and those that compose the clavicles are formed though an intramembranous process involving condensing mesenchymal stem cells that derive from the neural crest and are already resident in the developing skull. These precursor cells differentiate directly into osteoblasts apparently without a requirement for angiogenesis (17). This distinct difference in the development programs and embryological origin of the bone precursor cells responsible for endochondral and intramembranous bone is the most likely explanation for the site-specific skeletal phenotype of the Vhl-deficient mice. 

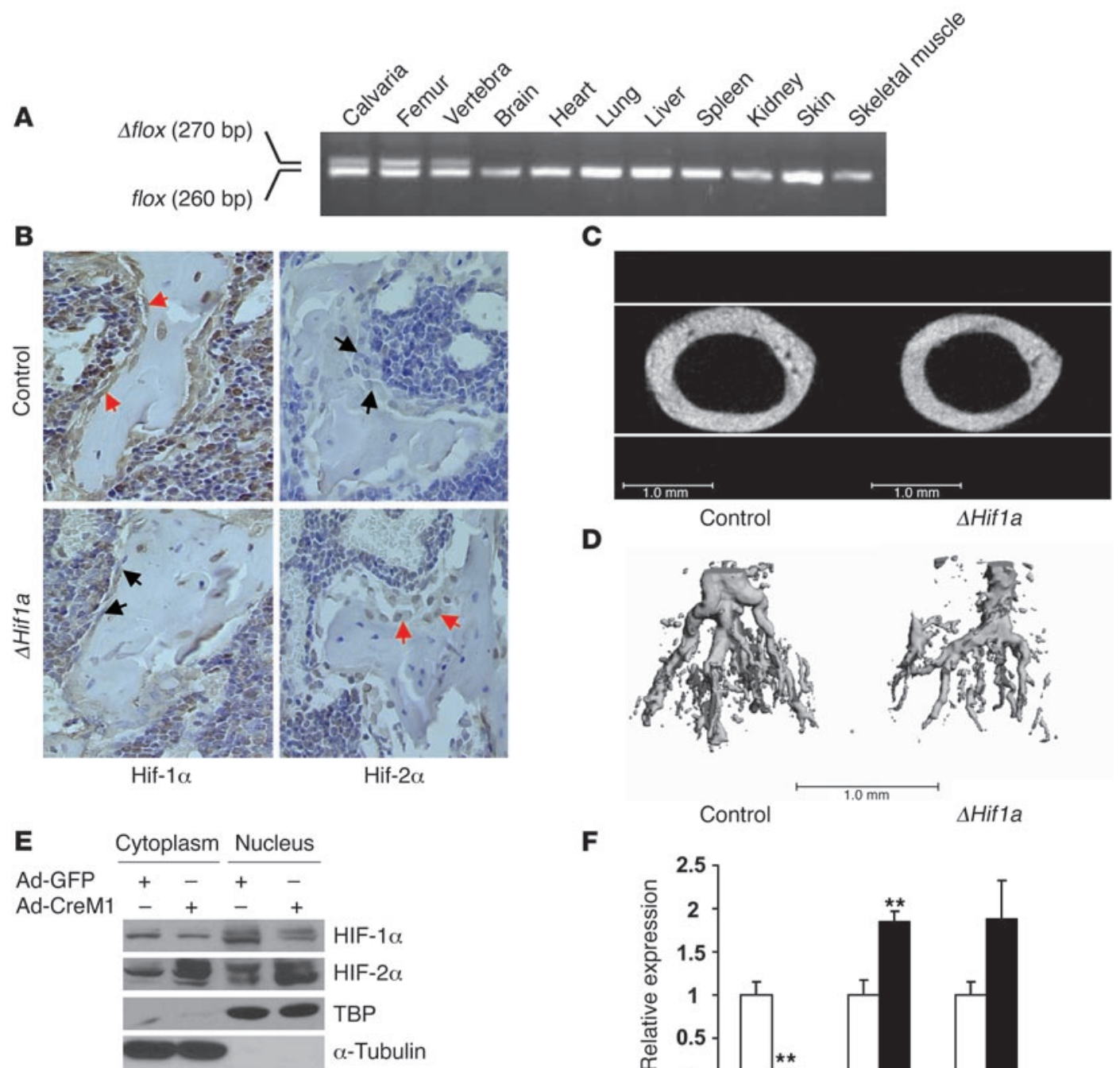

$\mathbf{F}$

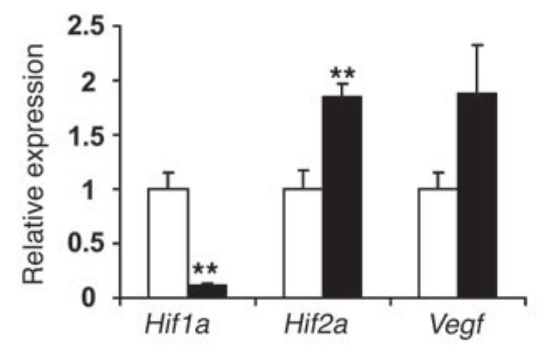

Figure 7

Mice lacking Hif1a in osteoblasts have narrow, poorly vascularized long bones. (A) PCR analysis of Cre-mediated recombination in selected tissues from a $\Delta$ Hif1a mouse. The recombined allele ( $\Delta$ flox) was present exclusively in bone tissue. (B) Representative histological sections of distal femurs from 6-week-old control and $\Delta$ Hif1a mice after staining with antibodies against HIF-1 $\alpha$ (left) or HIF-2 $\alpha$ (right) as described in Methods. Sections were counterstained with hematoxylin. Red arrows indicate positive and black arrows negative staining in osteoblasts. Original magnification, $\times 400$. (C) Representative images of femoral cross sections from control and $\Delta$ Hif1a mice. Scale bars: $1.0 \mathrm{~mm}$. (D) Representative $\mu C T$ images of vasculature in Microfil-perfused femurs from 3-week-old $\Delta$ Hif1a and control mice. Scale bar: $1.0 \mathrm{~mm}$. (E and F) Confluent monolayers of Hif1a floxed primary osteoblasts were infected with either Ad-GFP or Ad-CreM1 (100 MOI). (E) Proteins in the cytoplasm and nucleus were extracted separately 48 hours after infection. Immunoblotting analysis was performed with antibodies against HIF-1 $\alpha$ and HIF-2 $\alpha$. Immunoblots for TBP and $\alpha$-tubulin were used as loading controls for nuclear and cytoplasmic proteins, respectively. (F) Total mRNA was extracted from confluent monolayers of osteoblasts 48 hours after infection. Hif1a, Hif2a, and Vegf mRNA expression was determined by quantitative real-time PCR. ${ }^{* \star} P<0.01$.

Our studies provide additional insight into the functional relationship between HIF-1 $\alpha$ and HIF- $2 \alpha$. Osteoblast-specific deletion of Hifla produced undervascularized, thinner bones, whereas overexpression of both HIF- $1 \alpha$ and HIF- $2 \alpha$ by loss of $V h l$ yielded dramatically thicker, heavily vascularized bones. However, overexpressing only HIF- $2 \alpha$ in the osteoblasts by deleting both Hifla and Vbl phenocopied the vascular effects seen in the Vhl mutants but did not produce the same amount of bone. This suggests some degree of specificity for different HIF $\alpha$ proteins in these 2 tissues; i.e., it is possible that HIF- $1 \alpha$ and HIF- $2 \alpha$ act redundantly to promote angiogenesis, but they act differently with respect to inducing osteogenesis per se. Though HIF- $1 \alpha$ and HIF- $2 \alpha$ are homologous and both contain the conserved ODD domain (18), several previous studies suggest that HIF-1 $\alpha$ and HIF- $2 \alpha$ are not entirely redundant. For example, there are distinct differences in the expression levels of HIF- $1 \alpha$ and HIF- $2 \alpha$ in different cell types $(19,20)$. In addition, mice globally deficient in either Hifla or Hif $2 a$ die at different times during development, further suggesting that HIF- $1 \alpha$ and HIF- $2 \alpha$ have distinct functions in development (21-23). Moreover, the sets of genes regulated by these transcription factors, while overlapping, are not identical. For example, HIF- $1 \alpha$ appears to be more important than HIF- $2 \alpha$ with regard to regulation of hypoxiainducible glycolytic genes such as Pgk1, whereas HIF- $2 \alpha$ is a predominant regulator of hypoxic Vegf and erythropoietin (Epo) (24-26). Taken together, the data suggest that HIF- $1 \alpha$ and HIF-2 $\alpha$ have evolved to perform distinct functions in selected tissues but in certain contexts can substitute for one another.

Although a number of functions have been ascribed to the PVHL protein ligase, several lines of evidence including the studies described here suggest that its primary role is to regulate the destruction of HIF $\alpha$ subunits. Mice lacking $V h l$ in liver, growth plate, thymus, colon, and myeloid cells all demonstrate increased HIF $\alpha$ levels, increased expression of Vegf, and increased angiogenesis, underscoring the importance of $\mathrm{pVHL}$ as a negative regulator 
A

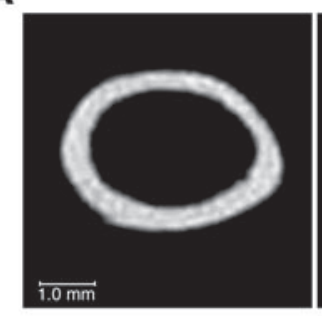

Control

C

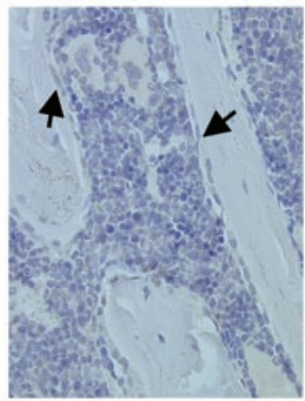

Control

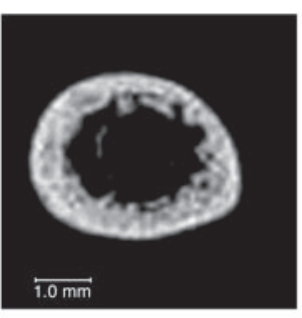

$\Delta$ Vhl/sHif1a

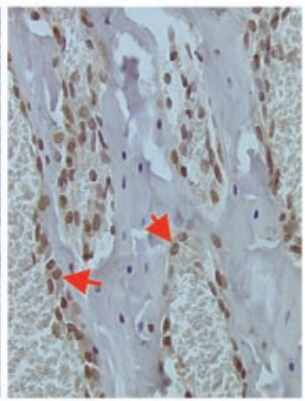

$\Delta$ Vhl/LHif1a
B
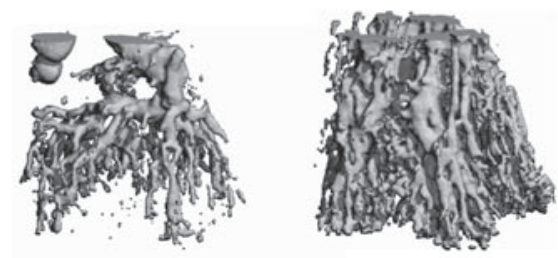

Control

D

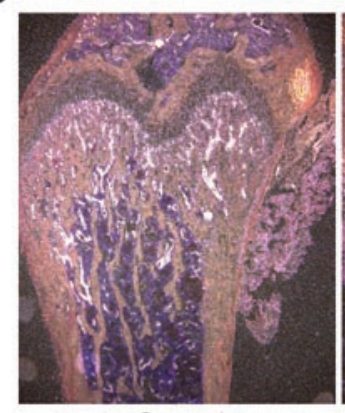

Control

\section{$\Delta$ Vhl/LHif1a}

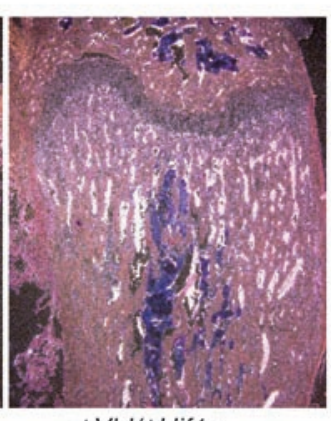

$\Delta V h l / \Delta H i f 1 a$

Figure 8

Bone and vascular phenotype in $\mathrm{Vhl}$ and Hif1a double-knockout mice. (A) Representative $\mu \mathrm{CT}$ images of femoral cross sections from 6-week-old $\Delta V h / / \Delta H i f 1$ a double mutant mice and wild-type controls. Scale bars: $1.0 \mathrm{~mm}$. (B) Representative images of Microfil-perfused femurs from 3-week-old $\Delta V h / / \Delta H i f 1$ a double-mutant mice and control littermates. Scale bar: $1.0 \mathrm{~mm}$. (C) Immunohistochemical analysis of HIF-2 $\alpha$ level in femoral sections from 6-week-old $\Delta$ Vhl/ $\Delta$ Hif1a double-mutant mice and control littermates. Sections were counterstained with hematoxylin. Red arrows indicate positive and black arrows negative staining in osteoblasts. Original magnification, $\times 400$. (D) In situ hybridization analysis with Vegf mRNA on histological sections from 6-week-old control and double-mutant femurs. Original magnification, $\times 100$.

of hypoxia-inducible genes in vivo (16, 27-30). Furthermore, a recent study using conditional expression of HIF- $1 \alpha$ and HIF- $2 \alpha$ variants that cannot be modified by prolyl hydroxylation showed that simultaneous expression of these stable HIF $\alpha$ molecules in liver and skin caused pathological changes that were indistinguishable from those seen when $V h l$ was deleted. In addition, activation of HIF- $2 \alpha$ (with or without HIF- $1 \alpha$ activation) induced expression of a set of genes that were also upregulated upon loss of $\mathrm{Vhl}$, suggesting that dysregulation of HIF target genes is primarily responsible for the pathological changes seen after $V h l$ inactivation in skin and liver (31). Therefore, even though pVHL is capable of binding other cellular proteins and possibly exerts functions separate from its role as an HIF $\alpha$ E3 ligase $(32,33)$, it appears that its primary role is to control cellular levels of HIF $\alpha$. Consequently, we attribute the skeletal effects seen in the Vhl mutant mice described in this article to increased HIF $\alpha$ activity rather than to HIF-independent phenomena.

Finally, our results enable the construction of a model for the role of the HIF $\alpha$ pathway in bone formation. In this model, osteoblasts residing on the nascent bone surface sense reduced oxygen or nutrient levels and upregulate HIF $\alpha$ subunits. Elevated HIF $\alpha$ transactivates target genes such as Vegf, which then stimulate new blood vessel formation and invasion into bone. This process is exponential, with ever-increasing numbers of new blood vessels introducing more osteoblast progenitors, which then mature and function to form more individual bone formation units. We speculate that the initial wave of osteogenesis results from signals emanating from the circulation, although it is also possible that osteoblast progenitors could themselves arrive strain (7). Mice with osteoblast-specific inactivation of Vhl or Hifla were obtained by intercrossing OC-Cre mice with mice homozygous for a floxed $\mathrm{Vhl}$ allele (27) or homozygous for a floxed Hifla allele (9). To generate mice lacking both $\mathrm{Vbl}$ and Hifla in osteoblasts, we first crossed $\Delta H i f l a$ mice with mice homozygous for a floxed $V b l$ allele and then interbred the offspring for 3 generations. Genotyping was performed as previously described $(7,9,27)$. All procedures involving mice were approved by the University of Alabama at Birmingham Institutional Animal Care and Use Committee.

Skeletal phenotyping, histological analysis, and in situ bybridization. The intact right femur was scanned using $\mu \mathrm{CT}$ ( $\mu$ CT20; SCANCO Medical) to assess bone mass, density, geometry, and trabecular microarchitecture. Parameters computed from these data include trabecular thickness, number, separation, and connectivity at the distal femoral metaphysis and cortical thickness and cross-sectional area at the mid-diaphysis. Dynamic bone formation rate was measured by injection of 2 sequential doses of calcein $(8 \mathrm{mg} / 10 \mathrm{ml}$ sterile saline) delivered in a total of $0.25 \mathrm{ml} 2$ and 6 days prior to sacrifice. Mouse femur and calvaria were dissected, fixed in $10 \%$ buffered formalin, decalcified in $8 \% \mathrm{Na}_{2}$ EDTA, paraffin embedded, and stained with $\mathrm{H} \& \mathrm{E}$ using standard methods. For immunohistochemistry, antigen retrieval was performed by boiling in $10 \mathrm{mM}$ sodium citrate ( $\mathrm{pH} \mathrm{6.0)}$ for 5 minutes. Sections were incubated with antibodies against HIF-1 $\alpha$ (C-19; Santa Cruz Biotechnology Inc.), HIF- $2 \alpha$ (Novus Biologicals), and pVHL (BD Biosciences - Pharmingen). In situ hybridization was performed using complementary ${ }^{35}$ S-labeled riboprobes as described previously (9).

Imaging of blood vessels. Blood vessels in bone were imaged using $\mu \mathrm{CT}$. Specimens were prepared in accordance with previously described methods (40). Briefly, after animals were euthanized, the thoracic cavity was opened, and the 
inferior vena cava was severed. The vasculature was flushed with $0.9 \%$ normal saline containing heparin sodium $(100 \mathrm{U} / \mathrm{ml})$ at a pressure of approximately $100 \mathrm{mmHg}$ via a needle inserted into the left ventricle. The specimens were then pressure fixed with $10 \%$ neutral buffered formalin. Formalin was flushed from the vessels using heparinized saline, and the vasculature was injected with a radiopaque silicone rubber compound containing lead chromate (Microfil MV-122; Flow Tech). Samples were stored at $4^{\circ} \mathrm{C}$ overnight for contrast agent polymerization. Mouse femurs were dissected from the specimens and soaked for 4 days in $10 \%$ neutral buffered formalin to ensure complete tissue fixation. Tissues were subsequently treated for 48 hours in a formic acid-based solution, Cal-Ex II (Fischer Scientific), to decalcify the bone and facilitate image thresholding of the femoral vasculature from the surrounding tissues. Images were obtained using a high-resolution (16- $\mu \mathrm{m}$ isotropic voxel size) $\mu \mathrm{CT}$ imaging system ( $\mu$ CT40; SCANCO Medical). A threshold of 306 was initially chosen based on visual interpretation of thresholded 2D tomograms. Histomorphometric parameters including vessel volume, connectivity, number, thickness, separation, and degree of anisotropy were evaluated.

Fetal mouse metatarsal angiogenesis assay. This assay was performed following the established method as discussed previously (41). Briefly, E17.5 embryos were removed from timed-pregnant mice, and metatarsals were dissected. The isolated metatarsals were cultured in 24-well tissue culture plates in $150 \mu \mathrm{l}$ of $\alpha$-MEM supplemented with $10 \%$ heat-inactivated FBS and $1 \%$ of penicillin/streptomycin for 72 hours. Two hundred and fifty microliters of fresh medium were then replaced, and metatarsals were cultured for 14 days, with replacement of medium every 3 days. Explants were then fixed in zinc formalin for 15 minutes at room temperature and subsequently stained for CD31 using a rat polyclonal antiserum against mouse CD31 (BD Biosciences - Pharmingen). Cultures were performed in sextuplicate, and each complete experiment was repeated at least twice.

Primary osteoblast isolation and culture, bypoxic experiment, and adenovirus infection. Osteoblasts were isolated from calvaria of newborn mice by serial digestion in $1.8 \mathrm{mg} / \mathrm{ml}$ of collagenase type I (Worthington Biochemical Corp.) solution. Calvaria were digested in $10 \mathrm{ml}$ of digestion solution for 15 minutes at $37^{\circ} \mathrm{C}$ under constant agitation. The digestion solution was then collected, and the digestion was repeated an additional 4 times. Digestion solutions 3-5, which contained the osteoblasts, were pooled together. After centrifugation, osteoblasts were obtained and cultured in $\alpha$-MEM containing $10 \%$ FBS and $1 \%$ penicillin/streptomycin at $37^{\circ} \mathrm{C}$ in a humidified incubator supplied with $5 \% \mathrm{CO}_{2}$. To examine the effect of experimental hypoxia, cells were cultured under hypoxic conditions ( $2 \%$ oxygen) at different times during culture or maintained under normoxic conditions ( $21 \%$ oxygen). All endpoints measured in hypoxic cells were compared with those in cells kept under normoxic conditions. Monolayer osteoblasts were infected with control adenovirus (Ad-GFP) or Cre recombinase virus M1 (Ad-CreM1) (Vector Laboratories) at an MOI of 100 for most experiments. Osteoblasts were harvested after 48 hours. Total mRNA was extracted from infected osteoblasts for deletion validation by realtime PCR, and proteins were extracted for immunoblotting. The remaining cells were replated on 100-mm tissue culture plates and subjected to proliferation and apoptosis assays or on 6-well plates for the mineralization assay.

Osteoblast proliferation and apoptosis in vitro. Mouse primary osteoblasts were plated on 100-mm plates with the density of $10^{6}$ cells/plate, and cultured in $\alpha$-MEM supplemented with $10 \%$ FBS and $1 \%$ penicillin/streptomycin for 24 hours. Cells were then starved in $\alpha$-MEM with $1 \%$ FBS for 12 hours, followed by the replacement with $10 \%$ FBS in $\alpha$-MEM and cultured for an additional 12 hours. For the proliferation assay, $\operatorname{BrdU}(10 \mu \mathrm{M})$ was added to the culture medium 2 hours before harvesting. Cells were stained with fluorescent anti-BrdU antibody and 7-amino-actinomycin D following the manufacturer's instructions (BD Biosciences). For the apoptosis assay, annexin $\mathrm{V}-\mathrm{PE}$ (BD Biosciences) and 7-AAD were added for staining. Osteoblasts were analyzed by FACSCalibur (BD) and 20,000 events were collected.
$A L P$ and von Kossa staining. Osteoblasts were plated on 6-well plates with a density of $10^{5}$ cells/well and cultured in $\alpha$-MEM until they were confluent. Medium was then changed to osteogenic medium with the addition of $\beta$-glycerophosphate $(10 \mathrm{mM})$ and ascorbic acid $(50 \mu \mathrm{g} / \mathrm{ml})$ to $\alpha$-MEM. Osteoblasts were then cultured for 14 days, with the medium changed every 3 days. Histochemical staining for ALP activity in the cells was determined using naphthol AS-MX phosphate (Sigma-Aldrich) as a substrate and fast red TR salt as a coupler. Von Kossa staining was carried out by adding $3 \%$ silver nitrate solution to formalin-fixed cells and exposing cells to UV light. The deposits of calcium were demonstrated by the formation of black nodules. Densitometric analysis of ALP and von Kossa staining was performed using NIH ImageJ 1.36b (http:rsb.info.nih.gov/ij/).

Quantitative real-time PCR. Total RNA was extracted from osteoblasts using the TRIzOL method as recommended by the manufacturer (Invitrogen). The yield and purity of RNA was estimated spectrophotometrically using the A260/A280 ratio. Three micrograms of RNA were reverse transcribed into cDNA using SuperScript first-strand synthesis system (Invitrogen). One microliter of cDNA was subjected to PCR amplification using SYBR GREEN PCR Master Mix (Applied Biosystems) and sequence-specific primers ( $\mathrm{Vhl}, 5^{\prime}$-GCCTATTTTTGCCAACATCACA-3' and 5'-TCATTCTCTCTATGTGCTGGCTTT-3'; Hif1a, 5'-CAAGATCTCGGCGAAGCAA-3' and 5'-GGTGAGCCTCATAACAGAAGCTTT-3'; Hif2 $a, 5^{\prime}$-CAACCTGCAGCCTCAGTGTATC-3' and 5'-CACCACGTCGTTCTTCTCGAT-3'; Vegf, 5'-CCACGTCAGAGAGCAACATCA-3' and 5'TCATTCTCTCTATGTGCTGGCTTT-3'; Vegf120, 5'-GCGGATCAAACCTCACCAAA-3' and 5'-CTCGGCTTGTCACATTTTTC-3'; Vegf164, 5'-ACAGGACAAAGCCAGAAAAACAC-3' and 5'- GTTTAACTCAAGCTGCCTCGCCT-3'; Vegf 188, 5'-GCGGATCAAACCTCACCAAA-3' and 5'GAACAAGGCTCACAGTGAACGC-3'; Glut1, 5'-GGGCATGTGCTTCCAGTATGT-3' and 5'-ACGAGGAGCACCGTGAAGAT-3'; Runx2, $5^{\prime}$-ATGCTTCATTCGCCTCAC-3' and 5'-CTCACGTCGCTCATCTTG-3'; OC, 5'-TCTGCTCACTCTGCTGAC-3' and 5'-GGAGCTGCTGTGACATCC$3^{\prime} ; \beta$-actin, $5^{\prime}$-CCCAGAGCAAGAGAGG-3' and $5^{\prime}$-GTCCAGACGCAGGATG$\left.3^{\prime}\right)$. PCR reactions were performed in triplicate for each cDNA, averaged, and normalized to endogenous $\beta$-actin reference transcripts.

Immunoblotting analysis. Whole cell lysate was obtained by homogenizing cell monolayers with cell lysis buffer in the presence of a protease inhibitor cocktail (Roche). Proteins in the cytoplasm and nucleus were separated using NE-PER nuclear and cytoplasmic extraction reagents according to the manufacturer's instructions (Pierce). Thirty micrograms of protein extracts were loaded onto an SDS mini-PAGE system after concentrations were determined by the Bradford method. After electrophoresis, proteins were transferred to a PVDF membrane using a Bio-Rad semi-dry transfer system. Protein transfer efficiency and size determination were verified using prestained protein markers. Membranes were then blocked with $5 \%$ dry milk in Tris-buffered saline Tween-20 for 1 hour at room temperature and subsequently incubated overnight with primary antibodies at $4{ }^{\circ} \mathrm{C}$. Signals were detected using an HRP-conjugated secondary antibody and the SuperSignal West Femto Maximum Sensitivity Substrate (Pierce). Antibodies used were anti-pVHL (BD Biosciences - Pharmingen), anti-HIF-1 $\alpha$ (NB100-105; Novus Biologicals), anti-HIF-2 $\alpha$ (NB100-122; Novus Biologicals), anti-TATA box-binding protein (anti-TBP) (Abcam), and anti-tubulin (Santa Cruz Biotechnology Inc.).

Immunofluorescence and confocal microscopy. Immunofluorescence assays were performed as previously described (42). Briefly, primary osteoblasts were plated on lysine-treated glass coverslips placed in 6-well plates. After culturing for 24 hours under either normoxic or hypoxic conditions, the cells were fixed with $4 \%$ paraformaldehyde for 10 minutes at room temperature, permeabilized with $0.2 \%$ Triton X-100 for 2 minutes, and incubated overnight at $4{ }^{\circ} \mathrm{C}$ with anti-HIF-1 $\alpha$ (NB100-105; Novus Biologicals) or antiHIF-2 $\alpha$ (NB100-122; Novus Biologicals) antibodies at a dilution of 1:100. 
After washing, coverslips were incubated with Alexa Fluor 488-labeled goat anti-mouse or anti-rabbit IgG $(\mathrm{H}+\mathrm{L})$ secondary antibody (Molecular Probes; Invitrogen) for 1 hour and mounted with VECTASHIELD mounting medium with DAPI (Vector Laboratories). Fluorescence localization was detected by confocal microscopy with a laser scanning microscope (LSM-510; Zeiss). Images were obtained with the same confocal settings for each set of experiments, and no autofluorescence was detected at these settings. Controls in which primary or secondary antibodies were omitted showed no staining. Images were processed using Adobe Photoshop 7.0 software.

ELISA. VEGF, OC, OPG, and TRAP $5 \mathrm{~b}$ in mouse sera were assayed using an ELISA according to manufacturer's recommendations. ELISA kits used were mouse VEGF Quantikine ELISA kit (R\&D Systems), mouse OC EIA kit (Biomedical Technologies Inc.), mouse OPG assay kit (Biomedica), and mouse TRAP assay kit (IDS). Optical density was determined using a plate reader (BioTek).

Statistics. For statistical analysis and comparison of histomorphometric parameters in control and mutant mice, Mann-Whitney $U$ test was per-

1. Steinbrech, D.S., et al. 2000. VEGF expression in an osteoblast-like cell line is regulated by a hypoxia response mechanism. Am. J. Physiol. Cell Physiol. 278:C853-C860.

2. Semenza, G.L. 1999. Regulation of mammalian $\mathrm{O}_{2}$ homeostasis by hypoxia-inducible factor 1. Annu. Rev. Cell Dev. Biol. 15:551-578.

3. Semenza, G.L. 2001. Hypoxia-inducible factor 1: oxygen homeostasis and disease pathophysiology. Trends Mol. Med. 7:345-350.

4. Huang, L.E., Gu, J., Schau, M., and Bunn, H.F. 1998. Regulation of hypoxia-inducible factor 1alpha is mediated by an $\mathrm{O}_{2}$-dependent degradation domain via the ubiquitin-proteasome pathway. Proc. Natl. Acad. Sci. U. S. A. 95:7987-7992.

5. Kaelin, W.G., Jr. 2002. Molecular basis of the VHL hereditary cancer syndrome. Nat. Rev. Cancer. 2:673-682.

6. Kallio, P.J., et al. 1998. Signal transduction in hypoxic cells: inducible nuclear translocation and recruitment of the $\mathrm{CBP} / \mathrm{p} 300$ coactivator by the hypoxia-inducible factor-1alpha. $E M B O J$. 17:6573-6586.

7. Zhang, M., et al. 2002. Osteoblast-specific knockout of the insulin-like growth factor (IGF) receptor gene reveals an essential role of IGF signaling in bone matrix mineralization. J. Biol. Chem. 277:44005-44012.

8. Haase, V.H. 2005. The VHL tumor suppressor in development and disease: functional studies in mice by conditional gene targeting. Semin. Cell Dev. Biol. 16:564-574.

9. Schipani, E., et al. 2001. Hypoxia in cartilage: HIF-1alpha is essential for chondrocyte growth arrest and survival. Genes Dev. 15:2865-2876.

10. Trueta, J., and Trias, A. 1961. The vascular contribution to osteogenesis. IV. The effect of pressure upon the epiphysial cartilage of the rabbit. J. Bone Joint Surg. Br. 43-B:800-813.

11. Trueta, J., and Buhr, A.J. 1963. The vascular contribution to osteogenesis. V. The vasculature supplying the epiphysial cartilage in rachitic rats. J. Bone Joint Surg. Br. 45:572-581.

12. Lewinson, D., and Silberman, M. 1992. Chondroclasts and endothelial cells collaborate in the process of cartilage resorption. Anat. Rec. 233:504-514.

13. Gerber, H.P., and Ferrara, N. 2000. Angiogenesis and bone growth. Trends Cardiovasc. Med. 10:223-228.

14. Hauge, E.M., Qvesel, D., Eriksen, E.F., Mosekilde, L., and Melsen, F. 2001. Cancellous bone remodeling occurs in specialized compartments lined by cells expressing osteoblastic markers. J. Bone Miner. Res. 16:1575-1582.

15. Eriksen, E.F., Eghbali-Fatourechi, G.Z., and Khosla, S. 2007. Remodeling and vascular spaces in bone.
J. Bone Miner. Res. 22:1-6.

16. Pfander, D., et al. 2004. Deletion of Vhlh in chondrocytes reduces cell proliferation and increases matrix deposition during growth plate development. Development. 131:2497-2508.

17. Chung, U.I., Kawaguchi, H., Takato, T., and Nakamura, K. 2004. Distinct osteogenic mechanisms of bones of distinct origins. J. Orthop. Sci. 9:410-414.

18. Maxwell, P.H., et al. 1999. The tumour suppressor protein VHL targets hypoxia-inducible factors for oxygen-dependent proteolysis. Nature. 399:271-275.

19. Bardos, J.I., and Ashcroft, M. 2005. Negative and positive regulation of HIF-1: a complex network. Biochim. Biophys. Acta. 1755:107-120.

20. Akeno, N., Czyzyk-Krzeska, M.F., Gross, T.S., and Clemens, T.L. 2001. Hypoxia induces vascular endothelial growth factor gene transcription in human osteoblast-like cells through the hypoxiainducible factor-2alpha. Endocrinology. 142:959-962.

21. Iyer, N.V., et al. 1998. Cellular and developmental control of $\mathrm{O} 2$ homeostasis by hypoxia-inducible factor 1 alpha. Genes Dev. 12:149-162.

22. Compernolle, V., et al. 2002. Loss of HIF-2alpha and inhibition of VEGF impair fetal lung maturation, whereas treatment with VEGF prevents fatal respiratory distress in premature mice. Nat. Med. 8:702-710.

23. Tian, H., Hammer, R.E., Matsumoto, A.M., Russell, D.W., and McKnight, S.L. 1998. The hypoxia-responsive transcription factor EPAS1 is essential for catecholamine homeostasis and protection against heart failure during embryonic development. Genes Dev. 12:3320-3324.

24. Hu, C.J., Wang, L.Y., Chodosh, L.A., Keith, B., and Simon, M.C. 2003. Differential roles of hypoxiainducible factor 1alpha (HIF-1alpha) and HIF2alpha in hypoxic gene regulation. Mol. Cell. Biol. 23:9361-9374.

25. Raval, R.R., et al. 2005. Contrasting properties of hypoxia-inducible factor 1 (HIF-1) and HIF-2 in von Hippel-Lindau-associated renal cell carcinoma. Mol. Cell. Biol. 25:5675-5686.

26. Wang, V., Davis, D.A., Haque, M., Huang, L.E., and Yarchoan, R. 2005. Differential gene up-regulation by hypoxia-inducible factor-1alpha and hypoxiainducible factor-2alpha in HEK293T cells. Cancer Res. 65:3299-3306.

27. Haase, V.H., Glickman, J.N., Socolovsky, M., and Jaenisch, R. 2001. Vascular tumors in livers with targeted inactivation of the von Hippel-Lindau tumor suppressor. Proc. Natl. Acad. Sci. U. S. A. 98:1583-1588.

28. Biju, M.P., et al. 2004. Vhlh gene deletion induces Hif-1-mediated cell death in thymocytes. Mol. Cell. Biol. 24:9038-9047.
29. Karhausen,J., et al. 2004. Epithelial hypoxia-inducible factor- 1 is protective in murine experimental colitis. J. Clin. Invest. 114:1098-1106. doi:10.1172/ JCI200421086.

30. Cramer, T., et al. 2003. HIF-1alpha is essential for myeloid cell-mediated inflammation. Cell. 112:645-657.

31. Kim, W.Y., et al. 2006. Failure to prolyl hydroxylate hypoxia-inducible factor alpha phenocopies VHL inactivation in vivo. EMBO J. 25:4650-4662.

32. Ohh, M., et al. 1998. The von Hippel-Lindau tumor suppressor protein is required for proper assembly of an extracellular fibronectin matrix. Mol. Cell. 1:959-968.

33. Esteban-Barragan, M.A., et al. 2002. Role of the von Hippel-Lindau tumor suppressor gene in the formation of beta1-integrin fibrillar adhesions. Cancer Res. 62:2929-2936.

34. Khosla, S., and Eghbali-Fatourechi, G.Z. 2006. Circulating cells with osteogenic potential. Ann. N. Y. Acad. Sci. 1068:489-497.

35. Dominici, M., et al. 2004. Hematopoietic cells and osteoblasts are derived from a common marrow progenitor after bone marrow transplantation. Proc. Natl. Acad. Sci. U. S. A. 101:11761-11766.

36. Eghbali-Fatourechi, G.Z., et al. 2005. Circulating osteoblast-lineage cells in humans. N. Engl. J. Med. 352:1959-1966.

37. Colnot, C.I., and Helms, J.A. 2001. A molecular analysis of matrix remodeling and angiogenesis during long bone development. Mech. Dev. 100:245-250.

38. Deckers, M.M., et al. 2000. Expression of vascular endothelial growth factors and their receptors during osteoblast differentiation. Endocrinology. 141:1667-1674.

39. Midy, V., and Plouet, J. 1994. Vasculotropin/vascular endothelial growth factor induces differentiation in cultured osteoblasts. Biochem. Biophys. Res. Commun. 199:380-386.

40. Duvall, C.L., Robert, T.W., Weiss, D., and Guldberg, R.E. 2004. Quantitative microcomputed tomography analysis of collateral vessel development after ischemic injury. Am. J. Physiol. Heart Circ. Physiol. 287:H302-H310.

41. Yen, M.L., et al. 2005. Diosgenin induces hypoxiainducible factor-1 activation and angiogenesis through estrogen receptor-related phosphatidylinositol 3-kinase/Akt and p38 mitogen-activated protein kinase pathways in osteoblasts. Mol. Pharmacol. 68:1061-1073.

42. Liu, Z., Tang, Y., Qiu, T., Cao, X., and Clemens, T.L. 2006. A dishevelled-1/Smad1 interaction couples WNT and bone morphogenetic protein signaling pathways in uncommitted bone marrow stromal cells. J. Biol. Chem. 281:17156-17163. 